J. Lake Sci. (湖泊科学), 2018, 30(1): 150-156

DOI 10. 18307/2018. 0115

(c) 2018 by Journal of Lake Sciences

\title{
巢湖表层沉积物中多溴联苯醚的分布和污染源解析
}

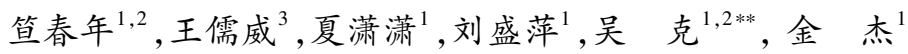 \\ ( 1 : 合肥学院生物与环境工程系, 合肥 230022) \\ ( 2 : 安徽省环境污染防治与生态修复协同创新中心, 合肥 230022) \\ (3: 中国科学技术大学地球与空间科学学院, 中国科学院壳幔物质与环境重点实验室,合肥 230026)
}

摘 要: 在巢湖不同区域采集了 16 个表层沉积物样品,利用气相色谱一质谱仪测定沉积物中低溴代多溴联苯醚 ( ${ }_{\mathrm{L}}$ PBDEs) 和 BDE209 的含量, 并对其残留、组成、空间分布和污染来源进行分析. 结果表明, 巢湖沉积物中共计检测到 9 种多溴联苯醚 (PBDEs) 化合物. $\Sigma_{\mathrm{L}}$ PBDEs 的含量为 $0.001 \sim 2.75 \mathrm{ng} / \mathrm{g}$, 平均值为 $1.15 \mathrm{ng} / \mathrm{g}$, 平均检测率为 $44.83 \%$; BDE209 的含量为 $1.16 \sim 5.49 \mathrm{ng} / \mathrm{g}$, 平均值为 $2.83 \mathrm{ng} / \mathrm{g}$, 检测率为 $100 \%$. 与国内其他区域相比, 巢湖沉积物中 PBDEs 含量 总体处于中等水平. 巢湖沉积物中各类 ${ }_{\mathrm{L}}$ PBDEs 和 BDE209 同系物含量为: 西半湖 >东半湖 > 湖心. 巢湖沉积物中 PBDEs 以 BDE209 为主要成分, 不同采样点 PBDEs 同系物的组成不同. 相关性分析表明 BDE209 与 ${ }_{\mathrm{L}}$ PBDEs 在环境中的迁移存在 相关性.

关键词 : 巢湖;多溴联苯醚;内源污染;源解析;浅水湖泊

\section{Distribution and sources of polybrominated diphenyl ethers in surface sediments of Lake Chaohu}

DA Chunnian ${ }^{1,2}$, WANG Ruwei ${ }^{3}$, XIA Xiaoxiao ${ }^{1}$, LIU Shengping ${ }^{1}$, WU Ke ${ }^{1,2 * *}$ \& JIN Jie ${ }^{1}$

(1: Department of Biology \&Environment Engineering, Hefei University, Hefei 230022, P.R. China)

(2: Cooperative Innovation Center of Environmental Pollution Prevention and Ecological Rehabilitation in Anhui Province, Hefei 230022 , P.R.China)

(3: CAS Key Laboratory of Crust-Material and Environments, School of Earth and Space Sciences, University of Science and Technology of China, Hefei 230026, P.R.China)

Abstract : 16 surface sediments were collected from Lake Chaohu. Low brominated polybrominated diphenyl ethers $\left({ }_{\mathrm{L}} \mathrm{PBDEs}\right)$ and BDE209 were measured by gas chromatography/mass spectrometry (GC/MS). The distributions, composition and sources of PBDEs were investigated. Results show that 9 PBDEs are detected. $\Sigma_{\mathrm{L}}$ PBDEs are in the range of 0.001 to $2.75 \mathrm{ng} / \mathrm{g}$ and 1.16 to 5.49 $\mathrm{ng} / \mathrm{g}$, with the average of 1.15 and $2.83 \mathrm{ng} / \mathrm{g}$, respectively. Compared with the other estuarine and coastal regions in China, PBDEs contents in Lake Chaohu are in the middle level. The spatial distribution of ${ }_{L}$ PBDEs and BDE209 contents follow the sequence of western half-lake> eastern half-lake> lake-central. BDE209 is the main component of PBDEs in the sediments of Lake Chaohu. In addition, the compositions of PBDEs homologues are different at different sampling sites. PBDEs are mainly derived from deca-

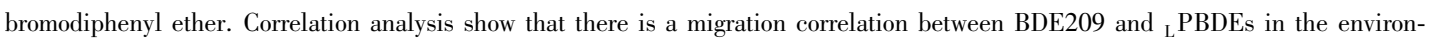
ment.

Keywords: Lake Chaohu; polybrominated diphenyl ethers; internal source pollution; source analysis; shallow lakes

多溴联苯醚 (简称 PBDEs) 在环境中具有持久性、生物蓄积性和长距离迁移等特点, 对生物和人体产生

* 安徽省自然科学基金项目(1608085MD78)、国家海洋局近岸域生态环境重点实验室资助项目 (201802)、安徽省教 育厅重点基金项目 (KJ2015A201，KJ2015A183，KJ2017A546) 和 2016 年高校优秀青年人才支持计划重点项目 (GXYQZD2016274) 联合资助. 2016-12-23 收稿; 2017-05-04 收修改稿. 笪春年 (1979 ), 女, 博士, 讲师; E-mail: 32768952@qq.com.

** 通信作者; E-mail: dachunnian2005@163.com. 
危害, 曾广泛应用于塑料、纺织、电器设备如电视机、电脑等物品的阻燃剂中 ${ }^{[1]}$. 尽管含 PBDEs 的商品生产 和使用已经被限制, 且逐渐被退出全球市场, 但近年来, 它们仍然在大气、水体、沉积物、土壤、植物、海洋以 及人体组织中被检测到 ${ }^{[2]}$. 有研究表明 PBDEs 在环境及人体中的含量呈现不断增加的趋势 ${ }^{[3]}$. 国内学者对 PBDEs 的研究在 2003 年开始逐渐兴起, 如分别有学者对青岛近岸沉积物、珠江三角洲沉积物以及珠江河口 水生生物中 PBDEs 的含量和分布进行了研究, 并在 PBDEs 的环境分布、迁移和归宿方面取得了一定的研究 成果 ${ }^{[1,4-5]}$.

巢湖是我国的五大淡水湖之一, 位于安徽省中部区域 ${ }^{[6]}$. 巢湖水产资源丰富, 生态效应极为敏感. 近几 十年来, 随着工农业的快速发展和人口规模的增加, 不同污染源的污染物通过土壤径流和大气降水等多种 途径进人巢湖水体, 使水环境日趋恶化, 水生环境受到威胁和破坏 ${ }^{[7]}$. 目前关于巢湖水体中的研究主要集中 在重金属污染和水体富营养化方面, 对于 PBDEs 的研究相对较少, 而 PBDEs 作为环境中持久性有机污染物 之一, 广泛存在于空气、水体和底泥、土壤等环境介质中 ${ }^{[8]}$. 由于其较高的亲脂性, 进人环境介质中的 PBDEs 易分配吸附到到生物体和沉积物中, 并通过食物链富集, 对人类健康和生态环境具有很大的潜在危害 ${ }^{[9-10]}$.

\section{1 材料与方法}

\section{1 样品采集}

于 2015 年 9 月使用抓斗采样器在巢湖西半湖 ( 6 个)、湖心 ( 4 个) 和东半湖 ( 6 个) 共采集 16 个表层沉 积物 ( $0 \sim 5 \mathrm{~cm})$ 样品 (图 1). 采集的样品迅速带回实验室在 $-20^{\circ} \mathrm{C}$ 温度下储存, 直至分析.

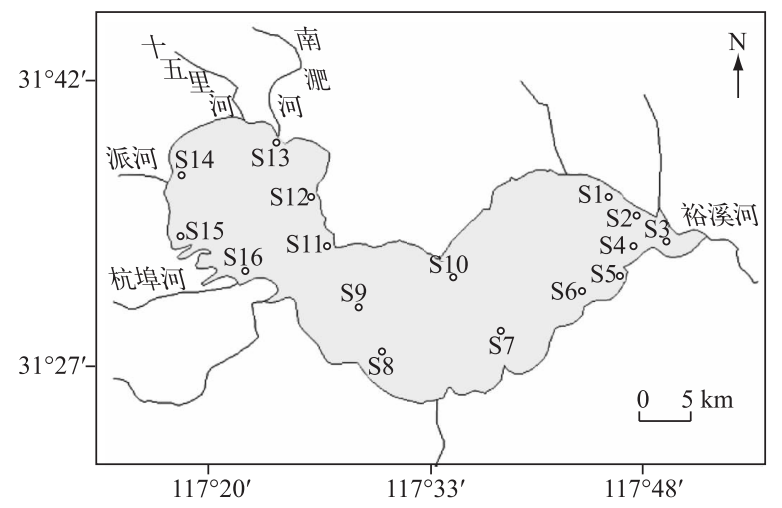

图 1 巢湖表层沉积物采样点分布

Fig.1 Distribution of the sampling sites in Lake Chaohu

\section{2 样品前处理}

样品提取前经冷冻干燥 $48 \mathrm{~h}$ 、磨碎、过 100 目篮后保存在深色磨口瓶中. 使用索氏抽提法对样品中的目 标物进行提取, 具体步骤为: 精确称量 $10 \mathrm{~g}$ 样品放人 $200 \mathrm{ml}$ 烧瓶中, 并加人体积比 $1: 1$ 混合的丙酮 -正已烷 溶剂、3 种 ${ }^{13} \mathrm{C}$ 标记的 PBDEs 回收率指示物和 $\mathrm{Cu}$ 片 (脱硫作用), 在 $45^{\circ} \mathrm{C}$ 水浴锅中抽提 $48 \mathrm{~h}$. 抽提结束后, 将 烧瓶中的抽提液在旋转蒸发仪上浓缩至 $1 \mathrm{ml}$, 浓缩液过多层硅胶氧化铝柱净化, 净化柱用 $35 \mathrm{ml}$ 正已烷、70 $\mathrm{ml}$ 的正已烷一二氯甲烷 (体积比 $1: 1$ ) 淋洗, 淋洗液旋转蒸发浓缩至 $1 \mathrm{ml}$, 转人细胞瓶中, 密闭保存, 仪器分析 前添加内标物为 BDE-71 (Accustandard Inc., USA).

\subsection{PBDEs 的测定}

PBDEs 的测定采用 Aglient 7890 气相色谱仪和 5975C 质谱仪, 色谱柱为 DB-5MS 毛细管柱 $(30 \mathrm{~m} \times 0.25$ $\mathrm{mm} \times 0.1 \mu \mathrm{m})$. 升温程序为: $60^{\circ} \mathrm{C}$ 下保留 $2 \mathrm{~min}$, 然后以 $10^{\circ} \mathrm{C} / \mathrm{min}$ 的速率升至 $200^{\circ} \mathrm{C}$ 并保持 $2 \mathrm{~min}$, 再以 $20^{\circ} \mathrm{C} /$ $\min$ 升到 $300^{\circ} \mathrm{C}$ 保持 $10 \mathrm{~min}$. BDE209 的测定采用 Thermo Trace Ultra 气相色谱仪和 Thermo DSQ II 质谱仪分 析, 色谱柱为 DB-5HT 毛细管柱 $(15 \mathrm{~m} \times 0.25 \mathrm{~mm} \times 0.1 \mu \mathrm{m})$, 升温程序为: 起始温度 $120^{\circ} \mathrm{C}$, 保持 $1 \mathrm{~min}$, 以 $25^{\circ} \mathrm{C} / \mathrm{min}$ 的速度升到 $330^{\circ} \mathrm{C}$, 保持 $10 \mathrm{~min}$. 载气均为高纯氮气, 离子源温度为 $150^{\circ} \mathrm{C}$. 界面温度 $280^{\circ} \mathrm{C}$. 选择 
离子为 79 和 81 . 选择离子监测模式 (SIM) 检测, $1 \mu \mathrm{l}$ 不分流进样.

\section{4 质量保证与质量控制}

每分析 5 个样品做 1 个加基样品 (检测实验方法的可靠性)、 1 个空白样品 (检测实验过程中外界因素 是否有干扰) 和 3 个平行样品 (检测实验方法的误差). 仪器检测限定义为 3 倍信噪比 ( $\mathrm{S} / \mathrm{N}$ ), 方法检测限范 围为 $0.001 \sim 0.004 \mathrm{ng} / \mathrm{g}$, 加基样品的回收率为 $98.6 \% \sim 106 \%$; 空白样品中均未检测到目标物质的存在, 平行 样品的相对标准偏差范围为 $0.3 \% \sim 8.0 \%$, 所有质量保证和质量控制均处于可接受的范围内.

\section{2 结果与讨论}

\section{1 沉积物中 PBDEs 含量}

在研究的 39 种低溴代 PBDEs $\left({ }_{\mathrm{L}} \mathrm{PBDE}\right)$ 和它们的主要同系物 BDE209 中, 仅检测到 BDE35、BDE37、 BDE47、BDE99、BDE85、BDE154、BDE153、BDE183 和 BDE2099 种化合物, 其他同系物均未被检测出. $\sum_{\mathrm{L}}$ PBDEs 的含量为 $0.001 \sim 2.75 \mathrm{ng} / \mathrm{g}$, 平均值为 $1.15 \mathrm{ng} / \mathrm{g}$, 平均检测率为 $44.83 \%$, BDE209 的含量为 $1.16 \sim$ $5.49 \mathrm{ng} / \mathrm{g}$, 平均值为 $2.83 \mathrm{ng} / \mathrm{g}$, 检测率为 $100 \%$ (表 1). 表明这类污染物在巢湖沉积物中有着普遍的分布. 在 检测到的目标化合物中,BDE209 和 BDE183 的检测率达到 100\%. 目前还没有关于 PBDEs 的沉积物标准限 值, 为了更好地掌握本研究中沉积物的 PBDEs 污染现状, 将研究结果与国内外最近的报道进行比较, 结果显 示, 巢湖表层沉积物中 PBDEs 的污染大致与国际上报道较清洁的淡水沉积物中的含量 ( $\sum$ PBDEs 含量为 $0.06 \sim 3.97 \mathrm{ng} / \mathrm{g}$ ) 处于同一水平 ${ }^{[11]}$.

表 1 巢湖表层沉积物中 ${ }_{\mathrm{L}}$ PBDEs 及 BDE209 的含量 *

Tab.1 Contents of ${ }_{\mathrm{L}}$ PBDEs and BDE209 in surface sediments of Lake Chaohu

\begin{tabular}{|c|c|c|c|c|}
\hline 目标化合物 & 最小值/(ng/g) & 最大值/(ng/g) & 平均值 $/(\mathrm{ng} / \mathrm{g})$ & 检测率/\% \\
\hline BDE35 & nd & 0.09 & 0.01 & 25.00 \\
\hline BDE37 & nd & 0.10 & 0.02 & 25.00 \\
\hline BDE47 & nd & 0.69 & 0.24 & 43.75 \\
\hline BDE99 & nd & 0.49 & 0.24 & 50.00 \\
\hline BDE85 & nd & 0.55 & 0.12 & 37.50 \\
\hline BDE154 & nd & 0.07 & 0.04 & 31.25 \\
\hline BDE153 & nd & 0.22 & 0.17 & 81.25 \\
\hline BDE183 & 0.001 & 0.55 & 0.32 & 100.00 \\
\hline BDE209 & 1.16 & 5.49 & 2.83 & 100.00 \\
\hline$\sum_{\mathrm{L}}$ PBDEs & 0.001 & 2.75 & 1.15 & 44.83 \\
\hline
\end{tabular}

* nd 表示未检出.

\section{2 沉积物中 PBDEs 的空间分布}

将 16 个采集点按照东半湖 ( 6 个点)、西半湖 (6个点)、湖心 ( 4 个点) 进行了分类比较 (图 2), 结果发现 各类 ${ }_{\mathrm{L}}$ PBDEs 和 BDE209 同系物含量为: 西半湖>东半湖>湖心. 西半湖沉积物中 ${ }_{\mathrm{L}}$ PBDEs 和 BDE209 含量较 高, 可能是由于西半湖人湖河流较多 (如: 派河、丙子河、塘西河等), PBDEs 随着市政和工业废水的排放进入 城市的地表径流, 流人巢湖西半湖; 而湖心沉积物中 ${ }_{\mathrm{L}}$ PBDEs 和 BDE209 同系物含量较低可能是由于湖心离 人湖的河口较远, 污染物随着湖水的稀释和扩散,其中浓度到湖心后大大减小.

\section{3 与其他地区沉积物中 PBDEs 的对比}

与国内其他区域相比, 巢湖沉积物中 $\sum_{\mathrm{L}} \mathrm{PBDEs}$ 的总含量处于中等水平, 与中国青岛近岸海域和东海海 域沉积物中 $\Sigma_{\mathrm{L}} \mathrm{PBDEs}$ 的残留水平基本相当, 但高于黄河人海口、莱州湾、长江三角洲和渤海沉积物中 $\sum_{\mathrm{L}} \mathrm{PBDEs}$ 的残留水平 (表 2), 可能的原因是黄河人海口、莱州湾等区域生态环境受到政府的保护, 外来污染 源进人研究区较少, 比如: 黄河人海口、莱州湾区域早在 1980 年就被政府授予国家级自然保护区 ${ }^{[10]}$. 巢湖沉 积物中 $\sum_{\mathrm{L}} \mathrm{PBDEs}$ 的总含量水平远远低于东江、珠江、太湖和珠江口沉积物中 $\sum_{\mathrm{L}} \mathrm{PBDEs}$ 的残留水平, 可能是 
因为珠江口、江苏等沿海区域工业经济发展快于安徽, 含 PBDEs 的电子产品和塑料产品使用量比安徽更多、 使用时间更早. 与国外研究区域相比, 巢湖沉积物中的 $\sum_{\mathrm{L}} \mathrm{PBDEs}$ 含量要高于美国的夏厄沃西河和萨吉诺 河, 但远远低于美国的 Hadley 湖和荷兰的 Scheldt 河, 这可能与当地经济发展水平相关. 另外, 本研究区沉积 物中的 BDE209 含量远远低于上述地区的 BDE209 含量 (表 2). 由此可知, 巢湖沉积物中 PBDEs 处于较低的 污染水平.

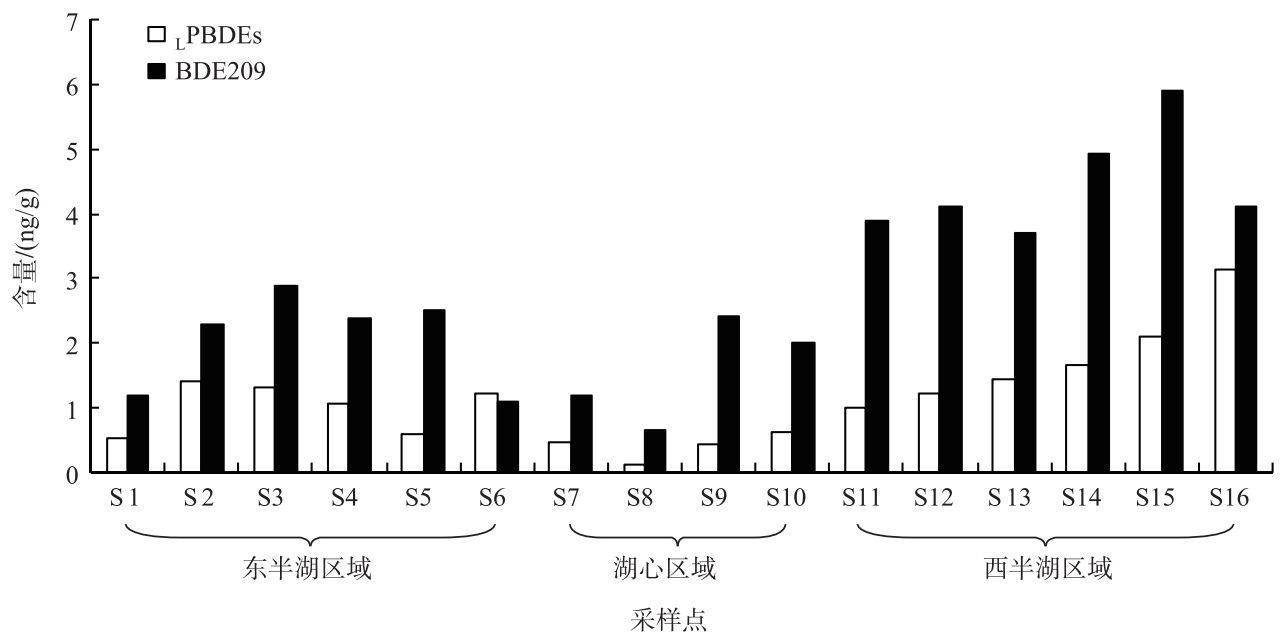

图 2 巢湖表层沉积物中 ${ }_{\mathrm{L}}$ PBDEs 和 BDE209 含量的空间分布

Fig.2 Distribution of ${ }_{\mathrm{L}}$ PBDEs and BDE209 contents in surface sediments of Lake Chaohu

表 2 与其他地区表层沉积物中 ${ }_{\mathrm{L}}$ PBDEs 和 BDE209 含量的对比

Tab.2 Comparison of ${ }_{\mathrm{L}}$ PBDEs and BDE209 contents in surface sediments of other areas around the world

\begin{tabular}{lccc}
\hline 采样地点 & $\Sigma_{\mathrm{L}} \mathrm{PBDEs} /(\mathrm{ng} / \mathrm{g})$ & $\mathrm{BDE} 209 /(\mathrm{ng} / \mathrm{g})$ & 参考文献 \\
\hline 珠江口(中国) & 3.10 & 18.50 & {$[1]$} \\
东江珠江(中国) & 26.92 & 1440.65 & {$[12]$} \\
黄河人海口(中国) & 0.69 & 2.79 & {$[13]$} \\
长江三角洲(中国) & 0.15 & 13.40 & {$[14]$} \\
青岛近岸(中国) & 1.40 & 37.49 & {$[4]$} \\
太湖(中国) & 5.21 & 5.10 & {$[15]$} \\
莱州湾(中国) & 0.32 & 6.40 & {$[17]$} \\
东海(中国) & 1.60 & 7.00 & {$[18]$} \\
渤海(中国) & 0.48 & 2.28 & {$[19]$} \\
夏厄沃西河(美国) & 0.56 & 4.76 & {$[20]$} \\
萨吉诺河(美国) & 0.61 & 28.80 & {$[21]$} \\
Hadley 湖(美国) & 13.90 & 22.00 & 本研究 \\
Scheldt 河(荷兰) & 24.00 & 2.83 & \\
巢湖(中国) & 1.15 & & {$[19]$} \\
\hline
\end{tabular}

\section{4 沉积物中 PBDEs 同系物的分布特征及来源}

本研究调查发现, 巢湖沉积物中的 PBDEs 以 BDE209 为主, 经过计算, BDE209 平均含量占 PBDEs 总量 的 71.14\% . 据相关文献报道 ${ }^{[5]}$, BDE209 是十溴联苯醚商品的主要成分 (成分含量 $>90 \%$ ), 我国生产的溴代 阻然剂成分以十溴联苯醚商品为主, 关于巢湖沉积物中多溴联苯醚以 BDE209 为主要成分的研究结果与我 国目前使用溴代阻然剂的现状相吻合, 也与现有文献中报道的关于我国沉积物中 PBDEs 的污染状况一 
致 $^{[11,13-14]}$. 巢湖不同采样点沉积物中 ${ }_{\mathrm{L}}$ PBDEs 的组成明显不同. 沉积物中 BDE183 在 16 个采样点均检测出, 而且在每个采样点所占的比重都较高. BDE183 是八溴联苯醚阻燃剂的主要成分, 这表明八溴联苯醚阻燃剂 在巢湖流域有一定程度的使用. BDE99、BDE47、BDE153、BDE85 和 BDE154 是 ${ }_{\mathrm{L}}$ PBDEs 的主要同系物, 也是 五溴联苯醚阻然剂的主要成分, 表明它们可能来自五溴联苯醚阻燃剂. 这些化合物在不同采样点均有不同 程度检测出, 尤其是 BDE153 的检测率达到 81.25\%, 只在湖心 3 个点 (S8、S9 和 S10) 未检测出 (图 3 ).

不同采样点 PBDEs 同系物的组成不同, 可能与巢湖流域 PBDEs 的来源有关. ${ }_{2}$ PBDEs 具有较高的大气 压和水溶解度, 易于通过空气及水输送 ${ }^{[22]}$, 因此巢湖区域 ${ }_{\mathrm{L}} \mathrm{PBDEs}$ 可能是通过远距离的大气或水体迁移而 来, 另外, 高溴代 PBDEs 在长距离的迁移过程中, 也可能产生脱溴作用形成 ${ }_{\mathrm{L}} \mathrm{PBDEs}{ }^{[23]}$. 高溴代 PBDEs 具有 低的挥发性和较高的辛醇-水分配系数, 更易吸附于细小颗粒物并随它们在环境中迁移 ${ }^{[24-25]}$, 因此也可推断 巢湖沉积物中 PBDEs 可能随颗粒物的迁移而来.

为了进一步识别巢湖沉积物中 PBDEs 的污染源, 运用 SPSS 统计软件对各研究区域 BDE209 与 ${ }_{\mathrm{L}}$ PBDEs 进行了相关分析 (表 3). 从 BDE209 与 BDE47、BDE199 和 BDE183 间均存在显著的相关性可以看出, 虽然 BDE209 与其他 PBDEs 同系物分别来自不同的溴代阻燃剂, 但 BDE209 与 BDE47、BDE199 和 BDE183 同系 物具有相同的输人途径, 它们可能是通过水体中颗粒物输人的, 它们之间较高的相关性是 PBDEs 在水体颗 粒物中再分配的结果. BDE37、BDE153、BDE154 和 BDE183 间也存在显著的相关性, 表明这几种多溴联苯醚 的环境行为和来源相近.

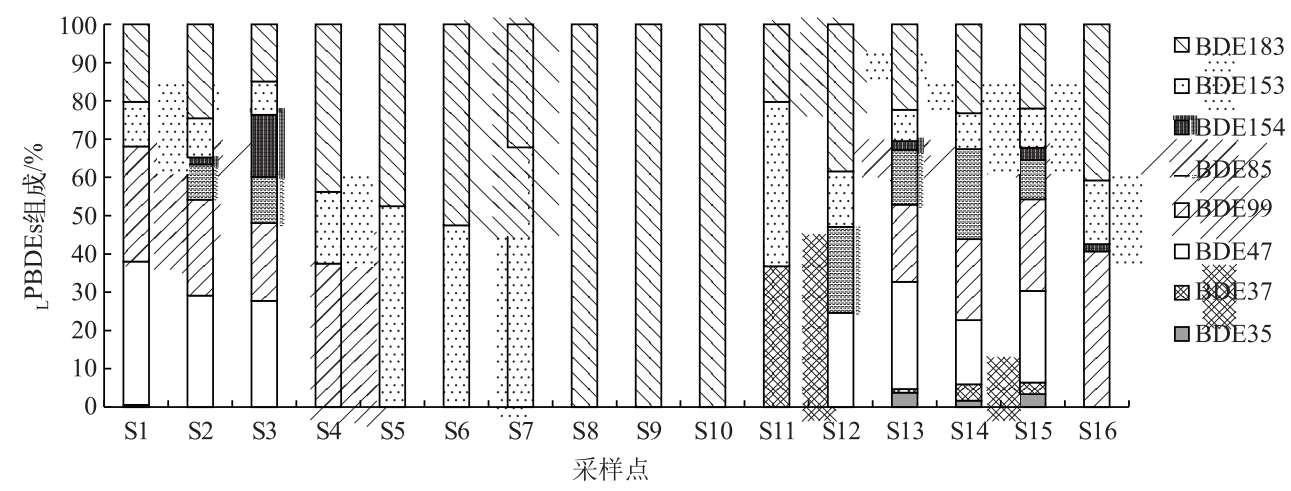

图 3 巢湖表层沉积物中 ${ }_{\mathrm{L}}$ PBDEs 的组成

Fig.3 Composition of ${ }_{\mathrm{L}}$ PBDEs in surface sediments of Lake Chaohu

表 3 PBDEs 间的 Pearson 相关性分析

Tab.3 Pearson correlation coefficients between PBDE congeners

\begin{tabular}{|c|c|c|c|c|c|c|c|c|c|}
\hline & BDE35 & BDE37 & BDE47 & BDE99 & BDE85 & BDE154 & BDE153 & BDE183 & BDE209 \\
\hline BDE35 & 1 & & & & & & & & \\
\hline BDE37 & 0.215 & 1 & & & & & & & \\
\hline BDE47 & -0.318 & -0.495 & 1 & & & & & & \\
\hline BDE99 & -0.011 & -0.449 & $0.445^{*}$ & 1 & & & & & \\
\hline BDE85 & 0.121 & -0.067 & -0.406 & -0.337 & 1 & & & & \\
\hline BDE154 & 0.117 & $0.456^{*}$ & $-0.589^{* *}$ & -0.347 & -0.073 & 1 & & & \\
\hline BDE153 & 0.172 & $0.694^{* *}$ & $-0.712^{* *}$ & -0.378 & 0.084 & $0.655^{* *}$ & 1 & & \\
\hline BDE183 & 0.363 & $0.568^{* *}$ & $-0.810^{* * *}$ & $-0.516^{*}$ & 0.350 & $0.745^{* *}$ & $0.880^{\text {*** }}$ & 1 & \\
\hline BDE209 & -0.027 & -0.061 & $0.626^{* *}$ & $0.525^{*}$ & -0.185 & -0.365 & -0.422 & $-0.484^{*}$ & 1 \\
\hline
\end{tabular}

$* *$ 表示在 $a=0.01$ 水平上显著相关; $*$ 表示在 $a=0.05$ 水平上显著相关. 


\section{3 参考文献}

[ 1 ] Chen SJ, Mai BX, Zeng YP et al. Polybrominated diphenyl ethers (PBDEs) in surfical sediments of the Pearl River Delta and adjacent South China Sea. Acta Scienctiae Circumstantiae, 2005, 25(9): 1265-1271. [陈社军, 麦碧涃, 曾永平等. 珠江三角洲及南海北部海域表层沉积物多溴联苯醚的分布特征. 环境科学学报, 2005, 25(9): 1265-1271.]

[ 2 ] Zhang LF, Huang YR, Dong L. Pollution of polybrominated diphenyl ethers in China. Environmental Chemistry, 2010, 29 (5) : 787-795. [张利飞, 黄业茹, 董亮. 多溴联苯醚在中国的污染现状研究进展. 环境化学, 2010, 29(5): 787-795.]

[ 3 ] Schecter A, Pavuk M, Päpke O et al. Polybrominated diphenyl ethers (PBDEs) in US mothers' milk. Environmental Health Perspectives. 2003, 111(14): 1723.

[ 4 ] Yang YL, Pan J, Li Y et al. Persistent organic pollutants polychlorinated biphenyls and polybrominated diphenyl ethers in coastal sediments from Qingdao. Science Bulletin, 2003, 48(21): 2244-2251. [杨永亮, 潘静, 李悦等. 青岛近岸沉积 物中持久性有机污染物多氯芸和多溴联苯醚. 科学通报, 2003, 48(21): 2244-2251.]

[ 5 ] Xiang CH, Luo XJ, Yu M et al. Distribution of polybrominated diphenyl ethers in aquatic species from the Pearl River Estuary. Environmental Science, 2006, 27(9): 1732-1737. [向彩红, 罗孝俊, 余梅等. 珠江河口水生生物中多溴联苯 醚的分布. 环境科学, 2006, 27(9): 1732-1737.]

[ 6 ] Wang X, Xi B, Huo S et al. Polybrominated diphenyl ethers occurrence in major inflowing rivers of Lake Chaohu (China) : Characteristics, potential sources and inputs to lake. Chemosphere, 2013, 93( 8) : 1624-1631.

[ 7 ] He Y, Xu FL, He W et al. Progresses in the studies on trace organic contaminants in Lake Chaohu ecosystem. Asian Journal of Ecotoxicology, 2016, 11(2): 111-123. [贺勇, 徐福留, 何伟等. 巢湖生态系统中微量有机污染物的研究进 展. 生态毒理学报, 2016, 11(2): 111-123.]

[ 8 ] Marvin C, Waltho J, Jia J et al. Spatial distributions and temporal trends in polybrominated diphenyl ethers in Detroit River suspended sediments. Chemosphere, 2013, 91: 778-783.

[ 9 ] Lin HT, Li QL, Zhang G et al. Pollution characteristics and human exposure level of atmospheric polybrominated diphenyl ethers in 8 cities of China. Environmental Science, 2016, 37(1):10-15. [林海涛, 李琦路, 张干等. 中国 8 个城市大 气多溴联苯醚的污染特征及人体暴露水平. 环境科学, 2016, 37(1): 10-15.]

[10] Wang W, Zhou JL, Pei SW et al. Research progress on pollution of polybrominated diphenyl ethers in environment. Environmental Chemistry, 2014, 33(7) : 1084-1093. [王维, 周俊丽, 裴淑玮等. 多溴联苯醚在环境中的污染现状研究进 展. 环境化学, 2014, 33(7): 1084-1093.]

[11] Zhao GF, Zhou HD, Du M et al. PBDEs in sediments from 14 principal tributaries of Haihe River and their potential risk. Environmental Science, 2011, 32(7) : 2069-2073. [赵高峰, 周怀东, 杜苗等. 海河流域 14 条河流表层沉积物中多 溴联苯醚的分布特征. 环境科学, 2011, 32(7) : 2069-2073.]

[12] Wang P, Shang H, Li H et al. PBDEs, PCBs and PCDD/Fs in the sediments from seven major river basins in China: Occurrence, congener profile and spatial tendency. Chemosphere, 2016, 144: 13-20.

[13] Yuan Z, Liu G, Lam MH et al. Occurrence and levels of polybrominated diphenyl ethers in surface sediments from the Yellow River Estuary, China. Environmental Pollution, 2016, 212 : 147-154.

[14] Zhou Y, Yin G, Asplund L et al. A novel pollution pattern: Highly chlorinated biphenyls retained in black-crowned night heron (Nycticorax nycticorax) and Whiskered tern (Chlidonias hybrida) from the Yangtze River Delta. Chemosphere, 2016, 150: 491-498.

[15] Zhou P, Lin K, Zhou X et al. Distribution of polybrominated diphenyl ethers in the surface sediments of the Taihu Lake, China. Chemosphere, 2012, 88: 1375-1382.

[16] Pan X, Tang J, Li J et al. Polybrominated diphenyl ethers (PBDEs) in the riverine and marine sediments of the Laizhou Bay area, North China. Journal of Environmental Monitoring, 2011, 13(4) : 886-893.

[17] Li Y, Lin T, Chen Y et al. Polybrominated diphenyl ethers (PBDEs) in sediments of the coastal East China Sea: occurrence, distribution and mass inventory. Environmental Pollution, 2012, 171: 155-161.

[18] Pan X, Tang J, Li J et al. Levels and distributions of PBDEs and PCBs in sediments of the Bohai Sea, North China. Journal of Environmental Monitoring, 2010, 12(6) : 1234-1241. 
[19] Yun SH, Addink R, McCabe JM et al. Polybrominated diphenyl ethers and polybrominated biphenyls in sediment and floodplain soils of the Saginaw River watershed, Michigan, USA. Archives of Environmental Contamination and Toxicology, $2008, \mathbf{5 5}(1): 1-10$.

[20] Song W, Ford JC, Li A et al. Polybrominated diphenyl ethers in the sediments of the Great Lakes. 1. Lake Superior. Environmental Science \& Technology, 2004, 38(12) : 3286-3293.

[21] de Boer J, Wester PG, van der Horst A et al. Polybrominated diphenyl ethers in influents, suspended particulate matter, sediments, sewage treatment plant and effluents and biota from the Netherlands. Environmental Pollution, 2003, 122(1): 63-74.

[22] Eskenazi B, Chevrier J, Rauch SA et al. In utero and childhood polybrominated diphenyl ether (PBDE) exposures and neurodevelopment in the CHAMACOS study. Environmental Health Perspectives, 2013, 121 (2) : 257-262.

[23] Lu Z, Letcher RJ, Chu S et al. Spatial distributions of polychlorinated biphenyls, polybrominated diphenyl ethers, tetrabromobisphenol A and bisphenol A in Lake Erie sediment. Journal of Great Lakes Research, 2015, 41(3) : 808-817.

[24] Li Y, Chen L, Wen ZH et al. Characterizing distribution, sources, and potential health risk of polybrominated diphenyl ethers (PBDEs) in office environment. Environmental Pollution, 2015, 198: 25-31.

[25] Besis A, Botsaropoulou E, Voutsa D et al. Particle-size distribution of polybrominated diphenyl ethers (PBDEs) in the urban agglomeration of Thessaloniki, northern Greece. Atmospheric Environment, 2015, 104: 176-185. 